\section{PSICOLOGIA IBEROAMERICANA}

\section{Psicología lberoamericana}

ISSN: 1405-0943

psicología.iberoamericana@uia.mx

Universidad Iberoamericana, Ciudad de

México

México

Barajas Márquez, Miriam Wendolyn; González Forteza, Catalina Francisca; Cruz del Castillo, Cinthia;

Robles García, Rebeca

El significado psicológico de una ruptura de pareja significativa en jóvenes universitarios

Psicología Iberoamericana, vol. 20, núm. 2, julio-diciembre, 2012, pp. 26-32

Universidad Iberoamericana, Ciudad de México

Distrito Federal, México

Disponible en: http://www.redalyc.org/articulo.oa?id=133928816004

Cómo citar el artículo

Número completo

- Más información del artículo

- Página de la revista en redalyc.org

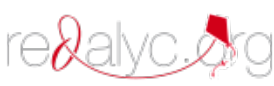

Sistema de Información Científica

Red de Revistas Científicas de América Latina, el Caribe, España y Portugal

Proyecto académico sin fines de lucro, desarrollado bajo la iniciativa de acceso abierto 


\title{
El significado psicológico de una ruptura de pareja significativa en jóvenes universitarios
}

\author{
The Psychological Meaning of a Meaningful Relationship \\ and Breakup in College Students
}

\author{
Miriam Wendolyn Barajas Márquez* \\ Universidad Nacional Autónoma de México \\ Rebeca Robles García \\ Catalina Francisca González Forteza \\ Instituto Nacional de Psiquiatría \\ Cinthia Cruz del Castillo \\ UNIVERSIDAD IBEROAMERICANA
}

\section{RESUMEN}

El objetivo del presente estudio fue obtener empíricamente el significado psicológico de una ruptura de pareja significativa en una muestra de jóvenes de la Universidad Iberoamericana, mediante la técnica de Redes Semánticas Naturales Modificadas (Reyes-Lagunes, 1993). Se encontró que el tamaño de la red para este estímulo fue igual a 176, y su núcleo se ubicó en las primeras 10 definidoras. Asimismo, se obtuvieron palabras con una connotación primordialmente negativa (p.e. tristeza, dolor, enojo), así como palabras asociadas a respuestas emocionales y conductuales desagradables. No obstante, también se encontraron definidoras con una connotación positiva (p.e. aceptación y crecimiento) que denotan un aprendizaje posterior a esta experiencia. Se concluye la necesidad de explorar más a fondo el significado de una ruptura importante dado el impacto que ésta puede tener sobre la salud física y mental del individuo, así como en la posibilidad de prevenir sus consecuencias negativas e incidir de manera efectiva a nivel clínico mediante intervenciones basadas en investigación realizada con población mexicana.

Palabras clave: pareja, jóvenes, ruptura amorosa, universitarios y redes semánticas.

\begin{abstract}
The objective of the present study was to empirically obtain the psychological meaning of a couple rupture in a sample of students of the Universidad Iberoamericana, by means of the technique of Modified Natural Semantic Networks (ReyesLagunes, 1993). The study found that the size of the network for this stimulus was equal to 176 and the nucleus located on the first ten defining. Also, words were obtained with a fundamentally negative connotation (ex. sadness, pain, angry) as well as associative words to emotional responses and dislikable behaviors. However, also found defining with a positive connotation (ex. acceptance and growth) that denotes learning subsequent to this experience. Concluding with the necessity to explore deeply the meaning of an important rupture given the impact that could have on the physical and mental health of the individual, as well as in the possibility of preventing negative consequences and influence in an effective way on clinical level by interventions based on investigation realized with Mexican population.

Keywords: couple, young people, breakup, college students and semantic networks.

* Nota de las autoras:

Agradecemos a todos los jóvenes que participaron en este estudio para apoyar a la presente investigación, la cual forma parte del Programa de Maestría y Doctorado en Psicología de la Universidad Nacional Autónoma de México. Este estudio se realizó gracias a la beca otorgada a la primera autora por el Consejo Nacional de Ciencia y Tecnología (Conacyt).

Para correspondencia e información respecto al presente artículo, favor de escribir a: miriamwendolyn@yahoo.com.
\end{abstract}




\section{INTRODUCCIÓN}

Durante los periodos de adolescencia y juventud, los seres humanos iniciamos relaciones interpersonales que marcan de forma considerable estos periodos Generalmente, una de las relaciones más importantes durante esta etapa es la de noviazgo, ya que permite al individuo, en términos ideales, cubrir necesidades que son esenciales para el desarrollo personal y social tales como la comunicación, el cariño, la confianza, la comprensión, entre otras (Becerril, 2001; Buss, 2005; Valdez-Medina, 2009).

Díaz-Loving y Sánchez (2004) definen a la pareja como la unión de dos seres humanos que viven ciertos pasos entrelazados en serie a través del tiempo, mismos que a su vez determinan el grado de intimidad y acercamiento que cada miembro percibe del otro. Esta relación se verá afectada tanto por las experiencias previas de cada uno, como por las expectativas que se tengan acerca de la relación (Collins \& Gillath, 2012).

Sin embargo, por tratarse de la interacción entre dos individuos con diferentes características personales, experiencias, actitudes y creencias, existe la posibilidad de que, en cualquier punto, esta relación entre en un conflicto y su dinámica se vea fracturada, llegando al punto de romper el vínculo amoroso, ya sea de manera temporal o permanente (Caruso, 2003; ValdezMedina, González-Amarratia, Torres \& Rocha, 2011).

Debido a la importancia que tiene una ruptura de noviazgo para los jóvenes, diversos autores se han ocupado de investigar las consecuencias de este evento en la salud física y mental de un individuo (Kloss \& Leissman, 2002; Park \& Blumberg, 2002; Ullrich \& Lutgendorf, 2002; Buss, 2005; Díaz-Loving, Valdez \& Pérez, 2005; Lewandowski \& Bizzoco, 2007; Collins \& Gillath, 2012).

En este sentido, las investigaciones realizadas reportan que las consecuencias que se experimentan con mayor frecuencia tras la pérdida de una pareja son: tristeza, llanto (Keller \& Nesse, 2005), enojo, inseguridad hacia uno mismo y celos hacia la ex pareja, disminución de la autoestima, un pobre autoconcepto (Locker, McIntosh, Hackney, Wilson \& Wiegand, 2010); depresión, ansiedad (Fisher, 2005; Donald, Dower, CorreaVelez \& Jones, 2006; Sbarra, 2006), altos niveles de estrés físico y emocional (Davis, Shaver \& Vernon; 2003) y conductas autodestructivas tales como las au- tolesiones, el consumo nocivo de alcohol y otras sustancias (Boelen \& Van de Bout, 2008; Perilloux \& Buss, 2008).

Sin embargo, aunque la mayoría de las investigaciones reportan resultados con una connotación primordialmente negativa, algunos autores han encontrado datos contrarios. Por ejemplo, Tashiro y Frazier (2003) hallaron un crecimiento personal importante posterior al duelo normal que sigue a la experiencia de la ruptura en aquellos participantes que obtenían puntajes altos en agradabilidad, motivación al crecimiento y que además contaban con buen apoyo social. Por su parte, Park, Bauer y Arbuckle (2009) asociaron ese mismo crecimiento a niveles altos de autoestima y a una tendencia por elaborar atribuciones internas ante los éxitos personales y atribuciones externas ante los fracasos.

Finalmente, es importante hacer notar que el efecto que tenga una ruptura de pareja en el individuo que la experimenta, ya sea positivo o negativo, dependerá de otros factores, tales como qué tan importante era esta relación de pareja, su duración, quién decidió romper, los motivos de ruptura, el grado de involucramiento, entre otros (Buss, 2005; Lewandowski \& Bizzoco, 2007; Waller, 2008; Locker et al., 2010).

No obstante, a pesar de la importancia que tiene para un individuo la ruptura de una relación significativa sobre su salud mental y su bienestar, no se ha explorado la manera en la que los jóvenes la definen. Por lo tanto, el objetivo del presente estudio fue obtener empíricamente el significado psicológico de dicho estímulo en una muestra de jóvenes universitarios.

\section{MÉTODO}

\section{Participantes}

La muestra estuvo integrada por 90 jóvenes estudiantes de Psicología de la Universidad Iberoamericana, campus Santa Fe, (65 mujeres y 26 hombres) con un rango de edad entre los 18 a los 29 años ( $M=20.50, D S=2.28)$. Dentro de la muestra, 51 jóvenes reportaron tener una pareja actualmentey 38 mencionaron no estar en una relación amorosa. De los participantes que tenían una pareja la media de duración en meses fue igual a 9.8 (DS=13.57). 


\section{Instrumento}

Se utilizó la técnica de Redes Semánticas Naturales Modificadas (RSNM) de Reyes Lagunes (1993), con el objetivo principal de identificar el significado psicológico de una "ruptura de una relación de pareja significativa". Esta técnica es principalmente exploratoria, se basa en una aproximación cualitativa, pero utiliza las matemáticas para su análisis, por lo que constituye una aproximación cuali-cuantitativa.

\section{Tipo de estudio}

Se llevó a cabo un estudio de campo de tipo transversal por medio de un muestreo no probabilístico e intencional.

\section{Procedimiento}

La técnica de las RSNM consiste en mostrar a los individuos algún estímulo desencadenador con el fin de evocar palabras ligadas o definidoras a dicho estímulo. De este modo, el instrumento se aplicó de manera grupal en los salones de clase de esta universidad; se explicó a los participantes el objetivo general y posteriormente se solicitó su participación voluntaria, garantizando la confidencialidad y anonimato de la información obtenida.

A cada participante se le entregó una hoja con el estímulo "ruptura de una relación de pareja significati- va" y se le indicó que definiera el concepto a través de por lo menos cinco palabras sueltas, sin utilizar artículos. Se les dio un minuto y otro adicional, en caso de que lo necesitaran. Posteriormente se pidió jerarquizar las palabras de acuerdo con su importancia, colocando el número 1 a la palabra que mejor definiera el concepto en cuestión, el 2 a la siguiente en importancia y así sucesivamente, hasta completar todas las palabras escritas previamente.

\section{RESULTADOS}

Una vez obtenida la información, se capturaron todas las palabras y su jerarquización en una hoja de cálculo del programa Excel ${ }^{\circ}$. Se codificó y se analizó la información, con lo que se obtuvieron los valores del tamaño de la red (TR), el peso semántico de cada palabra (PS), la distancia semántica cuantitativa (DSC) y el núcleo de la red (NR) conforme a la técnica RSNM propuesta por Reyes-Lagunes (1993).

De acuerdo con los resultados obtenidos, el tamaño de la red fue igual a 176. Se encontró que las definidoras más próximas al estímulo "ruptura de una relación de pareja significativa" fueron: 1) tristeza, 2) dolor, 3) enojo, 4) engaños y 5) infidelidad. Finalmente, el núcleo de la red, definido como la esencia del significado de un concepto, se encontró en las primeras 10 definidoras del estudio (palabras sombreadas en la tabla 1).

Tabla 1. Significado psicológico de una ruptura de pareja significativa

\begin{tabular}{|l|c|c|c|}
\hline Definidora & F & P.S. & D.S.C \\
\hline Tristeza & 59 & 472 & 100.00 \\
\hline Dolor & 42 & 323 & 68.43 \\
\hline Enojo & 41 & 265 & 56.14 \\
\hline Engaños & 26 & 153 & 32.42 \\
\hline Infidelidad & 22 & 140 & 29.66 \\
\hline Depresión & 20 & 137 & 29.03 \\
\hline Llanto & 19 & 122 & 25.85 \\
\hline Soledad & 18 & 121 & 25.64 \\
\hline Decepción & 16 & 107 & 22.67 \\
\hline Desconfianza & 14 & 99 & 20.97 \\
\hline Duelo & 11 & 92 & 19.49 \\
\hline
\end{tabular}

\begin{tabular}{|l|c|c|c|}
\hline Definidora & F & P.S. & D.S.C \\
\hline Desamor & 12 & 77 & 16.31 \\
\hline Desilusión & 11 & 75 & 15.89 \\
\hline Rencor & 14 & 71 & 15.04 \\
\hline Frustración & 10 & 67 & 14.19 \\
\hline Aprendizaje & 8 & 63 & 13.35 \\
\hline Monotonía & 9 & 60 & 12.71 \\
\hline Odio & 10 & 57 & 12.08 \\
\hline Pelea(s) & 9 & 56 & 11.86 \\
\hline Pérdida & 6 & 51 & 10.81 \\
\hline
\end{tabular}

f= Frecuencia, P.S= Peso semántico, D.S.C.= Distancia Semántica Cuantitativa. 
Por otra parte, en la tabla 2 se presentan algunas de las 176 palabras que se obtuvieron en esta investigación, las cuales, por su connotación, apoyan los hallazgos de otras investigaciones realizadas en torno al tema de la ruptura. De este modo se busca destacar los principales significados, por categorías, que dan los jóvenes de la muestra a la ruptura de una relación significativa. Finalmente se incluye un apartado en donde se muestran algunas palabras que (a excepción de la definidora "alcohol") no tienen una relación lógica aparente y que se pueden interpretar como parte de una experiencia personal en particular.

\section{Tabla 2. Definidoras agrupadas por significado}

\begin{tabular}{|c|c|}
\hline Definidoras asociadas con la tristeza* & Autores que han encontrado resultados similares: \\
\hline \multicolumn{2}{|l|}{ Tristeza* } \\
\hline Dolor* & Kloss \& Leissman, 2002; \\
\hline Depresión* & Park \& Blumberg, 2002; \\
\hline Llanto* & Ullrich \& Lutgendorf, 2002; \\
\hline Soledad* & Davis, Shaver \& Vernon, 2003; \\
\hline Decepción* & Fisher, 2005; \\
\hline Duelo* & \\
\hline Pérdida*** & Keller \& Nesse, 2005; \\
\hline Ansiedad *** & Donald, Dower, Correa-Velez \& Jones, 2006; \\
\hline Desesperación*** & Sbarra, 2006. \\
\hline \multicolumn{2}{|l|}{ Angustia*** } \\
\hline \multicolumn{2}{|l|}{ Estrés*** } \\
\hline Definidoras asociadas con el enojo* & Autores que han encontrado resultados similares: \\
\hline \multicolumn{2}{|l|}{ Enojo* } \\
\hline \multicolumn{2}{|l|}{ Rencor* ${ }^{*}$} \\
\hline Frustración** & Donald, Dower, Correa-Velez \& Jones, 2006; \\
\hline Odio** & Perilloux \& Buss, 2008; \\
\hline Coraje $^{* * *}$ & Locker, McIntosh, Hackney, Wilson \& Wiegand, 2010. \\
\hline \multicolumn{2}{|l|}{ Impotencia*** } \\
\hline \multicolumn{2}{|l|}{ Venganza*** } \\
\hline Motivos de ruptura & Autores que han encontrado resultados similares: \\
\hline \multicolumn{2}{|l|}{ Engaños* } \\
\hline \multicolumn{2}{|l|}{ Infidelidad* } \\
\hline \multicolumn{2}{|l|}{ Desconfianza* } \\
\hline \multicolumn{2}{|l|}{ Desamor** } \\
\hline Monotonía* * & Buss, 2005; \\
\hline Peleas** & Lewandowski \& Bizzoco, 2007; \\
\hline Celos*** & Waller, 2008; \\
\hline Incompatibilidad*** & Locker, McIntosh, Hackney, Wilson \& Wiegand, 2010; \\
\hline Maltratos*** & Valdez-Medina, González-Arratia, Torres \& Rocha, 2011. \\
\hline \multicolumn{2}{|l|}{ Aburrimiento*** } \\
\hline \multicolumn{2}{|l|}{ Intolerancia*** } \\
\hline \multicolumn{2}{|l|}{ Chismes*** } \\
\hline Abusos*** & \\
\hline
\end{tabular}




\begin{tabular}{|c|c|}
\hline Definidoras positivas & Autores que han encontrado resultados similares: \\
\hline \multicolumn{2}{|l|}{ Aprendizaje** } \\
\hline \multicolumn{2}{|l|}{ Entendimiento*** } \\
\hline Superación ${ }^{* * *}$ & \multirow{6}{*}{$\begin{array}{l}\text { Tashiro \& Frazier, 2003; } \\
\text { Díaz-Loving, Valdez \& Pérez, 2005; } \\
\text { Park, Bauer \& Arbuckle, } 2009 .\end{array}$} \\
\hline Aceptación*** & \\
\hline Reflexión*** & \\
\hline Crecimiento*** & \\
\hline \multirow{2}{*}{$\begin{array}{l}\text { Restablecimiento*** } \\
\text { Nuevas opciones*** }\end{array}$} & \\
\hline & \\
\hline Otras & Autores que han encontrado resultados similares: \\
\hline \multicolumn{2}{|l|}{ Alcohol $(1)^{* * *}$} \\
\hline \multicolumn{2}{|l|}{ Estafa*** } \\
\hline \multicolumn{2}{|l|}{$\operatorname{Edad}^{* * *}$} \\
\hline Familia*** & 'Boelen \& Van de Bout, 2008; \\
\hline Mujeres*** & Perilloux \& Buss, 2008. \\
\hline \multicolumn{2}{|l|}{ Ocuparse ${ }^{* *}$} \\
\hline \multicolumn{2}{|l|}{ Música*** } \\
\hline Religión*** & \\
\hline
\end{tabular}

* Palabras ubicadas en el núcleo de red, ** Palabras ubicadas dentro de las primeras veinte definidoras,

*** Palabras con menor peso semántico.

\section{DISCUSIÓN}

Como se pudo observar, la ruptura significativa de una relación de pareja suele ser un evento preponderantemente asociado con sentimientos y emociones negativas. De hecho, de las 176 palabras utilizadas por la muestra del estudio, sólo 20 se podrían considerar positivas (p.e. aceptación, resignación, aprendizaje, seguimiento, respeto y crecimiento) y la mayoría aluden a una recuperación, lo cual indica la necesidad de atravesar por un proceso de duelo y así poder alcanzar un crecimiento personal (Park, Bauer \& Arbuckle, 2009). Asimismo, resulta interesante destacar que ninguna de las definidoras consideradas como "positivas" se encontraron dentro del núcleo de la red. Por el contrario, las palabras ubicadas en primer lugar fueron la "tristeza", seguida de la palabra "dolor", así como otras definidoras relacionadas, como el "llanto", el "duelo", la "ansiedad" y la "depresión".

$\mathrm{Al}$ respecto, Caruso (2003) sostiene que una de las experiencias más dolorosas para el ser humano es la separación de la persona amada. Del mismo modo, Tashiro y Frazier (2003) consideran que una ruptura amorosa es una situación dolorosa que varía en intensidad, dependiendo de los factores que intervienen en ella y que si no se maneja adecuadamente puede llegar a tener consecuencias muy graves que van desde la tristeza y el enojo (dos de las definidoras ubicadas en el núcleo de la red), hasta desórdenes psicológicos tales como la ansiedad y la depresión (mismas que también mencionaron los participantes del estudio). Esto también concuerda con los hallazgos de Fisher (2005), quien asegura que existe un gran número de casos de ansiedad, depresión y suicidios ocasionados por el dolor de una ruptura amorosa.

En el caso del "llanto", al que se considera una manifestación conductual a consecuencia de una emoción primordialmente negativa, fue ubicado en el séptimo lugar de la lista; y de acuerdo con Keller y Nesse (2005), posee una función utilitaria de aviso hacia otras personas para solicitar ayuda, provocar empatía y fortalecer alianzas entre el afectado y aquellos que le pueden brindar apoyo y confort.

En tercer lugar se encontró la definidora "enojo" y se incluyeron en la lista otras palabras asociadas, es 
decir: rencor, venganza, frustración, odio e impotencia. Bajo esta misma línea, Lewandowski y Bizzoco (2007) reportaron una mayor cantidad de emociones negativas en aquellos individuos que habían terminado una relación en contra de su voluntad que en quienes habían decidido llevar a cabo la ruptura. Por su parte, Perilloux y Buss (2008) encontraron sentimientos de enojo, confusión, celos y baja autoestima, además de rumiación y pensamientos intrusivos en una muestra de jóvenes que habían sido blanco de la ruptura por parte de sus exparejas. Esto denota la importancia que tiene el papel que juega un individuo en dicho evento con respecto a quién inicia la ruptura y la necesidad de tomar en cuenta este factor a la hora de hacer un análisis más profundo del significado psicológico, ya que si bien la ruptura es una experiencia triste para ambos miembros de la pareja, no es de extrañar que resulte más traumatizante para quien no buscaba terminar con la relación en cuestión. Por lo tanto, es posible que quienes inician la separación se recuperen mucho más rápidamente de ésta que aquellos que terminan en contra de su voluntad (Locker et al., 2010; Collins \& Gillath, 2012).

Otro aspecto que resulta interesante destacar es que, a pesar de que no todas se hayan encontrado en el núcleo de la red, una importante cantidad de definidoras empleadas para definir el estímulo de interés se referían a los motivos por los que la relación pudo haber llegado a su fin (p.e. los engaños, la infidelidad, la desconfianza, la monotonía, los celos, los maltratos, los chismes, la falta de comunicación e incluso la falta de sexo). Esto coincide con los hallazgos reportados por
Buss (2005), quien señala como causas más frecuentes de una ruptura la infidelidad y el engaño (dos de las palabras que sí se ubicaron en el núcleo de la red), el retraimiento sexual, la crueldad (p.e. los abusos) y las faltas de respeto (p.e. los maltratos, las peleas y la intolerancia, encontrados en este estudio).

De los resultados de este estudio se puede concluir la complejidad del fenómeno y la necesidad de estudiarlo más a fondo tanto en los jóvenes como en otras poblaciones, tomando en cuenta el sexo, el tiempo transcurrido desde la ruptura, el número de parejas anteriores y otras variables que se han encontrado que guardan relación con este fenómeno, ya que por ejemplo, Díaz-Loving, Valdez y Pérez (2005) encontraron diferencias al respecto entre hombres y mujeres mexicanas. Las mujeres por su parte, reportaron sentimientos de tristeza, enojo y desconcierto, además de sentirse usadas y rechazadas; mientras que los hombres manifestaron sentimientos de malestar, decepción, humillación y enojo. Sin embargo, en el caso de la presente investigación no se tomó en cuenta para este análisis la diferenciación entre las definidoras utilizadas por los hombres y las que usaron las mujeres.

La importancia de explorar y definir a fondo la ruptura de una relación de pareja significativa radica en el impacto que puede llegar a tener sobre la salud física y mental de los individuos, así como en la posibilidad de prevenir sus consecuencias negativas o bien, de incidir de manera efectiva a nivel clínico mediante intervenciones basadas en investigaciones llevadas a cabo en población mexicana. 


\section{REFERENCIAS}

Becerril, D. (2001). Relaciones de pareja. Revista de Educación, 325, (49-56). Madrid, España.

Boelen, P. A. \& Van de Bout, J. (2008). Complicated grief and uncomplicated grief are distinguishable constructs. Psychiatry Research, 157, 311-314.

Buss, D. (2005). La evolución del deseo. España: Alianza Editorial.

Caruso, I. (2003). La separación de los amantes: Una fenomenología de la muerte. México: Siglo xxi.

Collins, T.J \& Gillath, O. (2012). Attachment, breakup strategies and associated outcomes: The effects of security enhancement on the selection of brealip strategies. Journal of Research in Personality, 46, 210-222.

Davis, S., Shaver, P.R., \& Vernon, M.L. (2003). Physical, emotional and behavioral reactions to breaking up: The roles of gender, age, emotional involvement and attachment style. Personality and Social Psychology Bulletin, 29(871-884).

Díaz-Loving, R. \& Sánchez, R. (2004). Psicología del amor: Una visión integral de la relación de pareja. México: Porrúa.

Díaz-Loving, R., Valdez, J. \& Pérez, M. (2005). Los hombres y las mujeres en México: Dos mundos distantes y complementarios. México: Porrúa.

Donald, M., Dower, J. Correa-Velez, I. \& Jones, M. (2006). Risk and protective factors for medically serious suicide attempts: A comparison of hospital based with population-based damples of young adults. Australian and New Zealand Journal of Psychiatry, 40, 87-96.

Fisher, H. (2005). Por qué amamos. México: Santillana.

Keller, M.C., \& Nesse, R.M. (2005). Is low mood an adaptation?: Evidence for subtypes with symptoms that match precipitants. Journal of Affective Disorders. 86, 27-35.

Kloss, J. \& Leissman, S. (2002). An exposure-based examination of the effects of written emotional disclosure. British Journal of Health. 7(1), 31-46.

Lewandowski, G.W. Jr, \& Bizzoco, N. (2007). Addition through subtraction: Positive outcomes following the loss of a dating relationship. Journal of Positive Psychology, 2(40-54).
Locker, L., McIntosh, W.D., Hackney, A.A., Wilson, J.H. \& Wiegand, K.E. (2010). The breakup of Romantic Relationships: Situational Predictors of Perception of Recovery. North American Journal of Psychology, 12(3), (565-578).

Park, C.L., \& Blumberg, C.J. (2002). Disclosing trauma through writing: Testing the meaning-making hypothesis. Cognitive Therapy and Research. 26, 597-616.

Park, S.W., Bauer, J.J. \& Arbuckle, N.B. (2009). Growth motivation attenuates the self-serving attribution. Journal of Research in Personality. 43, 914-917.

Perilloux, C. \& Buss, D. M. (2008) Breaking up romantic relationships. Costs experienced and coping strategies deployed. Evolutionary Psychology. 6(1), 164-181.

Reyes Lagunes, I. (1993). Las redes semánticas naturales, su conceptualización y su utilización en la construcción de instrumentos. Revista Mexicana de Psicología $y$ Personalidad. 9(1), 81-97.

Sbarra, D.A. (2006). Predicting the onset of emotional recovery following nonmarital relationship dissolution: Survival analyses of sadness and anger. Personality and Social Psychology Bulletin, 32(298-312).

Tashiro T, \& Frazier P. (2003). "I'll never be in a relationship like that again": Personal growth following romantic relationship breakups. Personal Relationships, 10(113-128).

Ullrich, P.M. \& Lutgendorf, S.K. (2002). Journaling about stressful events: Effects of cognitive processing and emotional expression. Annals of Behavioral Medicine. 24, 244-250.

Valdez-Medina, J.L. (2009). La búsqueda del equilibrio personal: Una nueva teoría que explica las causas del miedo y del sufrimiento y que nos enseña a combatirlos. México: EDAMEX.

Valdez-Medina, J.L., González-Arratia, N.I., Torres, M.A. \& Rocha, N.C. (2011). Estrategias biológicas y psicosocioculturales que intervienen en la conservación de la pareja: Un análisis por sexo. Enseñanza e Investigación en Psicología. Vol. 16 (1), 57-72.

Waller, K.L. (2008). Trait self-esteem moderates the effect of initiator status on emotional and cognitive responses to romantic relationship dissolution. Dissertation Abstracts International, 69, 19-77. 